\title{
DYNAMIC DEFORMATION OF AXIALLY SYMMETRIC ELASTIC MEMBRANES*
}

BY

R. W. DICKEY

University of Wisconsin

1. Introduction. The purpose of this paper is to discuss the dynamic behavior of various axially symmetric membranes, and in particular to find explicit solutions where possible, and numerical solutions where this not feasible.

In Sec. 2 of this paper dynamic equations are derived following the development in [1]. Thus the equations are formulated in Lagrange coordinates and the boundary conditions are taken on the known unstrained body. These equations are in general a pair of nonlinear wave equations with four characteristic directions. However, the possibility exists that these characteristics may become imaginary and the physical implications of this possibility are discussed.

One feature that recurs in the membrane approximation is the surprising difficulty of solving problems with small deformations. For example, in [2] great difficulty was encountered in finding numerical solutions for the slightly extended cylindrical membrane, while solutions for large extensions were found with relative ease. This phenomenon also occurs if a perturbation of the equations about an unstressed state is attempted. In this case a high order space derivative is lost from the equations and with it the ability to satisfy the prescribed boundary conditions. Corneliussen and Shield [3] circumvented this difficulty by considering a circular cylindrical membrane which was extended and inflated in such a way that the stressed state was again a circular cylinder. Linearizing the equations about this known finite deformation it is possible to find small amplitude vibrations. In Sec. 3 of this paper we develop a procedure to study the motion of pre-stressed membranes of Mooney and classical materials. Here two approaches are used: (1) a solution is found which is valid for small time (with no restriction on the magnitude of the deflections) and (2) a numerical solution is obtained for the exact equations.

2. The equations of motion. The physical situation to be considered is shown in Fig. 2.1. An axially symmetric elastic surface generated by rotating a curve $x=x_{0}(s)$, $r=r_{0}(s)$ ( $s$ is arc-length) about the $x$ axis is in an unstrained state at time $t=0$. For $t>0$ the surface is assumed to deform into another surface of revolution determined by $x=x(s, t), r=r(s, t)$; thus $s$ is a so-called Lagrangian parameter such that $x=$ $x_{0}(s, 0), r=r_{0}(s, 0)$ characterizes the initial position.

The strain is defined to be the ratio of the change in length of a line element to its original length. With this definition the strain $\epsilon_{a}$ in the axial direction is given by

$$
\epsilon_{a}=\left(\left(d x^{2}+d r^{2}\right)^{1 / 2}-d s\right) / d s=\left(x^{\prime 2}+{r^{\prime}}^{2}\right)^{1 / 2}-1,
$$

and the strain $\epsilon_{c}$ in the circumferential direction by

$$
\epsilon_{c}=\left(r d \theta-r_{0} d \theta\right) / r_{0} d \theta=r / r_{0}-1 .
$$

*Received December 16, 1966; revised manuscript received May 1, 1967. This paper represents results obtained at the Courant Institute of Mathematical Sciences, New York University, under the sponsorship of the U. S. Army Research Office-Durham, Contract DA-31-124-ARO-D-360. 


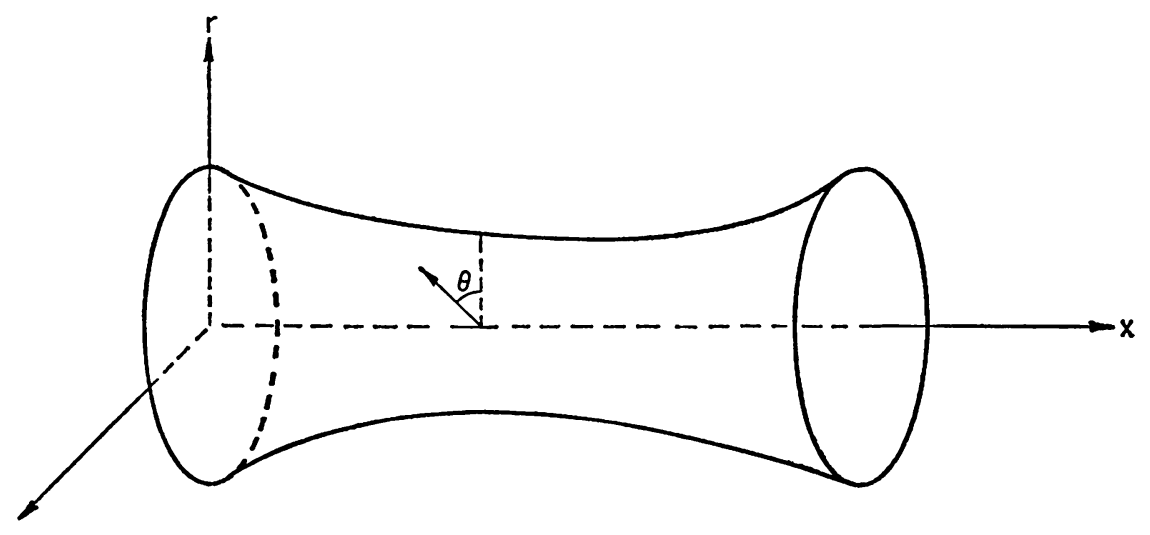

FIG. 2.1

The prime denotes differentiation with respect to the parameter $s$.

Elastic bodies are characterized here by the existence of a function $W=W\left(\epsilon_{a}, \epsilon_{c}\right)$. The function $W\left(\epsilon_{a}, \epsilon_{c}\right)$ is defined with respect to the undeformed body such that the potential (strain) energy of any state of deformation is given by

$$
\iiint_{V} W\left(\epsilon_{a}, \epsilon_{c}\right) d v
$$

$V$ being the undeformed, unstrained body. Since the membrane approximation assumes the strains to be constant through the thickness $h$, assumed uniform, and in addition axial symmetry has been required, the integral (2.3) can be written as

$$
2 \pi h \int_{0}^{e s} W\left(\epsilon_{a}, \epsilon_{c}\right) r_{0} d s .
$$

The kinetic energy of an element of the deformed body is

$$
(\bar{\rho} / 2)\left(x_{t}^{2}+r_{t}^{2}\right) d \bar{v}
$$

$\bar{\rho}$ being the density of the deformed volume element $d \bar{v}$. Since mass is conserved, it follows that

$$
\bar{\rho} d \bar{v}=\rho d v
$$

where $\rho$ is the density of the unstrained body (assumed constant). Thus the kinetic energy of the body is

$$
\iiint_{V} \frac{\rho}{2}\left(x_{t}^{2}+r_{t}^{2}\right) d v
$$

Again it is possible to perform two of the integrations in (2.5) to obtain

$$
2 \pi h \int_{0}^{e s} \frac{\rho}{2}\left(x_{t}^{2}+r_{t}^{2}\right) r_{0} d s .
$$

Hamilton's principle requires that the membrane move so that

$$
2 \pi h \int_{0}^{t} \int_{0}^{s s}\left\{\frac{\rho}{2}\left(x_{t}^{2}+r_{t}^{2}\right)-W\left(\epsilon_{a}, \epsilon_{c}\right)\right\} r_{0} d s d t
$$


is stationary. Thus the Euler equations (cf. [4]) for (2.7) become the equations of motion for the membrane. After substitution for $\epsilon_{a}$ and $\epsilon_{c}$ from (2.1) and (2.2) these Euler equations are easily found to be

$$
\begin{aligned}
\rho r_{0} x_{t \iota} & =\frac{\partial}{\partial s}\left(\frac{r_{0} x^{\prime}}{\left(x^{\prime 2}+r^{\prime 2}\right)^{1 / 2}} W_{\epsilon_{a}}\right), \\
\rho r_{0} r_{t \iota} & =\frac{\partial}{\partial s}\left(\frac{r_{0} r^{\prime}}{\left(x^{\prime 2}+r^{\prime 2}\right)^{1 / 2}} W_{\epsilon_{a}}\right)-W_{\epsilon_{c}} .
\end{aligned}
$$

These nonlinear partial differential equations have the form of nonlinear wave equations. Since initial value problems associated with them are to be treated, it is important to study their characteristics-in particular, it is desirable that the characteristics should be real (otherwise, the initial value problem would not be well posed, see [5, Chapter V] on hyperbolic equations). The characteristics are obtained from the following differential equation (see [5, pp. $171 \mathrm{ff}$.$] ):$

$$
\rho^{2} \phi_{t}^{4}-\rho\left[W_{\epsilon_{a} \epsilon_{0}}+\frac{W_{\epsilon_{a}}}{\left(x^{2}+r^{\prime 2}\right)^{1 / 2}}\right] \phi_{t}^{2} \phi_{s}^{2}+\frac{W_{\epsilon_{a}} W_{\epsilon_{a \epsilon_{a}}}}{\left(x^{\prime 2}+r^{\prime 2}\right)^{1 / 2}} \phi_{s}^{4}=0 .
$$

The equation may be factored to find the characteristic slopes

and

$$
(d s / d t)^{2}=W_{\epsilon_{a} \epsilon_{a}} / \rho
$$

$$
\left(\frac{d s}{d t}\right)^{2}=\frac{W_{\epsilon_{e}}}{\rho\left(x^{\prime 2}+r^{\prime 2}\right)^{1 / 2}} .
$$

Thus the question of the hyperbolicity of (2.8) depends on the nature of the strain energy density function $W$. For infinitesimal strains the strain energy density function for many materials would have the form of the classical strain energy density function

$$
W=\frac{\lambda}{2}\left(\epsilon_{a}+\epsilon_{c}\right)^{2}+\mu\left(\epsilon_{a}^{2}+\epsilon_{c}^{2}\right)
$$

as a limit case, with $\lambda$ and $\mu$ the two Lamé constants. In this case the partial derivatives of $W$ occurring in (2.10) and (2.11) are

$$
W_{\epsilon_{\mathrm{a}}}=(\lambda+2 \mu)\left(\epsilon_{a}+\epsilon_{c}\right), \quad W_{\text {ece. }}=\lambda+2 \mu .
$$

Thus the right hand side of (2.10) is always positive in this case. The right hand side of (2.11) is positive only if $W_{\epsilon_{0}}>0$, and this in turn will hold only if $\epsilon_{a}+\epsilon_{c}>0$. Since it is reasonable to expect the strain energy to increase as the strains increase, it will be assumed that $W_{\text {.. }}>0$ if the strains are positive. Thus Eqs. (2.8) are hyperbolic when the membrane is in tension.

The fact that Eqs. (2.8) may change type (cf. [6]) causes difficulty. For example, on physical grounds the initial boundary value problem seems a reasonable problem to impose on (2.8), i.e. an initial unstrained state is specified:

$$
\begin{array}{ll}
r(s, 0)=r_{0}(s), & r_{t}(s, 0)=0, \\
x(s, 0)=x_{0}(s), & x_{t}(s, 0)=0,
\end{array}
$$

with certain boundary conditions

$$
\begin{array}{ll}
r(0, t)=f_{1}(t), & r\left(s_{f}, t\right)=f_{2}(t), \\
x(0, t)=g_{1}(t), & x\left(s_{f}, t\right)=g_{2}(t) .
\end{array}
$$


A typical problem might be that the radii of the ends are fixed while the membrane is extended, in which case (2.15) would become

$$
\begin{array}{ll}
r(0, t)=a, & r\left(s_{f}, t\right)=b, \\
x(0, t)=g_{1}(t), & x\left(s_{f}, t\right)=g_{2}(t),
\end{array}
$$

where $a$ and $b$ are the radii of the ends. Equation (2.14) corresponds to the unstrained state, $\epsilon_{a}=\epsilon_{c}=0$ at $t=0$. Thus by (2.11) and (2.13), the initial data are given on a parabolic line (cf. [6]) of Eqs. (2.8). However, it will be assumed that no compressions develop if the ends are pulled apart (i.e. that $\epsilon_{a}+\epsilon_{c}$ is not negative).

The difficulties mentioned above may be avoided altogether if the surface is initially in tension rather than unstrained. In this case Eqs. (2.8) are definitely hyperbolic and the initial boundary value problem would be well posed. This approach was successfully used by Corneliussen and Shield [3] and will also be employed in Sec. 3 of this paper.

3. The dynamic equations. For classical materials (2.12) the equations of motion (2.8) become

$$
\begin{aligned}
& \rho r_{0} x_{t \iota}= \frac{\partial}{\partial s}\left\{\left[(\lambda+2 \mu) r_{0}+\frac{\lambda r-2(\lambda+\mu) r_{0}}{\left(x^{\prime 2}+r^{\prime 2}\right)^{1 / 2}}\right] x^{\prime}\right\} \\
& \rho r_{0} r_{t \iota}=\frac{\partial}{\partial s}\left\{\left[(\lambda+2 \mu) r_{0}+\frac{\lambda r-2(\lambda+\mu) r_{0}}{\left(x^{\prime 2}+r^{\prime 2}\right)^{1 / 2}}\right] r^{\prime}\right\} \\
& \quad+2(\lambda+\mu)-(\lambda+2 \mu) \frac{r}{r_{0}}-\lambda\left(x^{\prime 2}+{r^{\prime 2}}^{1 / 2} .\right.
\end{aligned}
$$

In the context of the present paper it is also reasonable to consider material described by the Mooney strain energy density function

$$
\begin{aligned}
W=C_{1}\left[\left(\epsilon_{a}+1\right)^{2}+\left(\epsilon_{c}+1\right)^{2}\right. & +\frac{1}{\left.\left(\epsilon_{a}+1\right)^{\frac{2}{2}\left(\epsilon_{c}+1\right)^{2}}\right]} \\
& +C_{2}\left[\left(\epsilon_{a}+1\right)^{2}\left(\epsilon_{c}+1\right)^{2}+\frac{1}{\left(\epsilon_{a}+1\right)^{2}}+\frac{1}{\left(\epsilon_{c}+1\right)^{2}}\right] .
\end{aligned}
$$

In this case Eqs. (2.8) become

$$
\begin{aligned}
& \rho r_{0} x_{t t}=2 \frac{\partial}{\partial s}\left\{r_{0} x^{\prime}\left(C_{1} \frac{r_{0}^{2}}{r^{2}}+C_{2}\right)\right. {\left.\left[\frac{r^{2}}{r_{0}^{2}}-\frac{1}{\left(x^{\prime 2}+r^{\prime 2}\right)^{2}}\right]\right\} } \\
& \rho r_{0} r_{t t}=2 \frac{\partial}{\partial s}\left\{r_{0} r^{\prime}\left(C_{1} \frac{r_{0}^{2}}{r^{2}}+C_{2}\right)\left[\frac{r^{2}}{r_{0}^{2}}-\frac{1}{\left(x^{\prime 2}+r^{\prime 2}\right)^{2}}\right]\right\} \\
&-2\left[C_{1}+C_{2}\left({x^{\prime 2}}^{2}+{r^{\prime}}^{2}\right)\right]\left[\frac{r}{r_{0}}-\frac{r_{0}^{3}}{r^{3}\left(x^{\prime 2}+r^{\prime 2}\right)}\right] .
\end{aligned}
$$

An infinite unstressed circular cylinder $x_{0}=s, r_{0}=a$ may be put into a state of stress by stretching each meridional arc-length element by an amount $\sigma$ while keeping the radius fixed. In this state the cylinder is described by $x=\sigma s, r=a$. This is not an equilibrium position for the membrane if no external pressure is applied; thus if initial conditions

$$
\begin{array}{ll}
x(s, 0)=\sigma s, & x_{t}(s, 0)=0, \\
r(s, 0)=a, & r_{t}(s, 0)=0
\end{array}
$$


are prescribed the membrane is in motion at some time $t>0$. This problem may be solved explicitly with a solution of the form

$$
x(s, t)=\sigma s, \quad r(s, t)=a f(t)
$$

where $f(0)=1$ and $d f(0) / d t=0$ to satisfy (3.3). If (3.4) is introduced into Eqs. (3.1) the solution for classical materials is found to be

$$
x=\sigma s, \quad r=a\left\{\frac{(\sigma-1) \lambda}{\lambda+2 \mu} \cos \left[\frac{1}{a}\left(\frac{\lambda+2 \mu}{\rho}\right)^{1 / 2} t\right]+\frac{(2-\sigma) \lambda+2 \mu}{\lambda+2 \mu}\right\} .
$$

The Eqs. (3.2) for Mooney materials reduce to the ordinary differential equation for $f$

$$
\frac{d^{2} f}{d t^{2}}+\frac{2\left(C_{1}+C_{2} \sigma^{2}\right)}{\rho a^{2} \sigma^{2}} \frac{\sigma^{2} f^{4}-1}{f^{3}}=0
$$

which does not lend itself to explicit solution because of its nonlinearity. It would be reasonable to expect the solution for the infinite cylinder to be a good description of the motion of a very long cylinder in its central portion.

For shorter cylinders this approximation is less valuable and it is necessary to introduce different techniques. As indicated in the introduction the usual perturbation methods fail, so that instead of searching for solutions valid for small deflections, a method will be developed below to find solutions valid for small time. Thus, while a perturbation technique attempts to find solutions valid for large time if the deflections are small, the small time approximation attempts to find solutions which are valid for large deflections if the time is small. To linearize the equations for small time the nonlinear coefficients are expanded in a power series in time. The coefficients of the various terms in the power series may be evaluated from the initial conditions and the differential equations. For small time the zero order term is assumed to be a good approximation and the prescribed initial boundary value problem is solved for these linear equations. At the end of this section the small time solution is compared with the numerical solution for the actual nonlinear equations, and very good agreement is found for a surprisingly long time.

To simplify the notation for the small time linearization of (3.1) introduce new variables

$$
\psi=\frac{\lambda+2 \mu}{\rho}+\frac{\lambda r-2(\lambda+\mu) a}{\rho a\left(x^{\prime 2}+r^{\prime 2}\right)^{1 / 2}}, \quad \phi=\left(x^{\prime 2}+{r^{\prime}}^{2}\right)^{1 / 2} .
$$

If the unstressed position of the membrane is a finite circular cylinder Eqs. (3.1) may be written

$$
\begin{aligned}
x_{t}-\frac{\partial}{\partial s}\left(\psi x^{\prime}\right) & =0 \\
r_{t}-\frac{\partial}{\partial s}\left(\psi r^{\prime}\right)+\frac{\lambda+2 \mu}{\rho a^{2}} r & =\frac{2(\lambda+\mu)}{\rho a}-\frac{\lambda}{\rho a} \phi .
\end{aligned}
$$

These equations are to be solved for a finite cylinder which is not initially in equilibrium, i.e. the initial values (3.3) are prescribed and in addition boundary values

$$
\begin{array}{ll}
x(0, t)=0, & x\left(s_{f}, t\right)=\sigma s_{f}, \\
r(0, t)=a, \quad r\left(s_{f}, t\right)=a .
\end{array}
$$


Assume the functions $\psi$ and $\phi$ may be expanded in a power series of the form

$$
\psi=\sum_{n=0}^{\infty} \psi_{n}(s) t^{n}, \quad \phi=\sum_{n=0}^{\infty} \phi_{n}(s) t^{n}
$$

at $t=0, \psi=\psi_{0}(s)$ and $\phi=\phi_{0}(s)$ or

$$
\psi_{0}(s)=\frac{\lambda+2 \mu}{\rho}+\frac{\lambda r(s, 0)-2(\lambda+\mu) a}{\rho a\left(x^{\prime}(s, 0)^{2}+r^{\prime}(s, 0)^{2}\right)^{1 / 2}}
$$

and

$$
\phi_{0}(s)=\left(x^{\prime}(s, 0)^{2}+r^{\prime}(s, 0)^{2}\right)^{1 / 2} .
$$

Thus $\psi_{0}(s)$ and $\phi_{0}(s)$ may be evaluated from the initial conditions (3.3). It is found that

$$
\psi_{0}=(\sigma-1)(\lambda+2 \mu) / \rho \sigma
$$

and

$$
\phi_{0}=\sigma .
$$

For small time it will be assumed that $\psi \approx \psi_{0}(s)$ and $\phi \approx \phi_{0}(s)$. Thus, replacing $\psi$ and $\phi$ in Eqs. (3.7) by $\psi_{0}$ and $\phi_{0}$, equations are obtained whose solutions are good for small time. The resulting equations are

$$
\begin{gathered}
x_{t t}-\frac{(\sigma-1)(\lambda+2 \mu)}{\rho \sigma} x^{\prime \prime}=0, \\
r_{t t}-\frac{(\sigma-1)(\lambda+2 \mu)}{\rho \sigma} \gamma^{\prime \prime}+\frac{(\lambda+2 \mu)}{\rho a^{2}} r=\frac{(2-\sigma) \lambda+2 \mu}{\rho a} .
\end{gathered}
$$

This same procedure may be carried through for Mooney materials (cf. (3.2)). The small time equations are found to be

$$
\begin{gathered}
x_{\imath}-\frac{2\left(C_{1}+C_{2}\right)\left(1-1 / \sigma^{4}\right)}{\rho} x^{\prime \prime}=0, \\
r_{t t}-\frac{2\left(C_{1}+C_{2}\right)\left(1-1 / \sigma^{4}\right)}{\rho} r^{\prime \prime}+\frac{2\left(C_{1}+C_{2} \sigma^{2}\right)}{\rho a^{2}} r=\frac{2\left(C_{1}+C_{2} \sigma^{2}\right)}{\rho a \sigma^{2}} .
\end{gathered}
$$

A Fourier series solution of (3.11) for the initial boundary value problem (3.3) and (3.8) is given by

$$
x=\sigma s, \quad r=\frac{4 \lambda \sigma}{a(\lambda+2 \mu) \pi} \sum_{n=0}^{\infty} \frac{\sin \alpha_{n}^{c} s \cos \beta_{n}^{c} t}{(2 n+1) \gamma_{n}^{c}}+A^{c} e^{\delta^{c} s}+B^{c} e^{-\delta^{c} s}-\frac{(\sigma-1) \lambda a}{\lambda+2 \mu}+a
$$

where

$$
\begin{gathered}
\alpha_{n}^{c}=\frac{(2 n+1) \pi}{s_{f}}, \quad \beta_{n}^{r}=\left[\frac{\lambda+2 \mu}{\rho}\left\{\frac{1}{a^{2}}+\frac{(2 n+1)^{2} \pi^{2}(\sigma-1)}{\sigma s_{f}}\right\}\right]^{1 / 2}, \\
\gamma_{n}^{c}=\frac{1}{a^{2}}\left(\frac{\sigma}{\sigma-1}\right)+\frac{(2 n+1)^{2} \pi^{2}}{s_{f}^{2}}, \quad \delta^{c}=\frac{1}{a}\left(\frac{\sigma}{\sigma-1}\right)^{1 / 2}, \\
A^{c}=\frac{(\sigma-1) \lambda a}{2(\lambda+2 \mu) \sinh \left(\delta^{c} s_{f}\right)}\left(1-e^{-\delta^{c} s_{f}}\right), \quad B^{c}=\frac{(\sigma-1) \lambda a}{2(\lambda+2 \mu) \sinh \left(\delta^{c} s_{f}\right)}\left(\exp \left(\delta^{c} s_{f}\right)+1\right) .
\end{gathered}
$$


Similarly the solution of (3.12) is

$$
\begin{aligned}
& x=\sigma s, \\
& r=\frac{4 \Lambda^{2} \delta^{m^{2}}}{\pi} \sum_{n=0}^{\infty} \frac{\sin \alpha_{n}^{M} s \cos \beta_{n}^{M} t}{(2 n+1) \gamma_{n}^{M}}+A^{M} \exp \left(\delta^{M} s\right)+B^{M} \exp \left(-\delta^{M} s\right)-\Lambda^{2}+a
\end{aligned}
$$

where

$$
\begin{gathered}
\Lambda^{2}=a\left(\sigma^{2}-1\right) / 2, \quad \alpha_{n}^{M}=(2 n+1) \pi / s_{f} \\
\beta_{n}^{M}=\left[\frac{2\left(C_{1}+C_{2} \sigma^{2}\right)}{\rho a^{2}}+\frac{2(2 n+1)^{2} \pi^{2}\left(C_{1}+C_{2}\right)\left(\sigma^{4}-1\right)}{\rho \sigma^{4} s_{f}^{2}}\right]^{1 / 2} \\
\delta^{M}=\frac{\sigma^{2}}{a}\left[\frac{C_{1}+C_{2} \sigma^{2}}{\left(C_{1}+C_{2}\right)\left(\sigma^{4}-1\right)}\right]^{1 / 2}, \quad \gamma_{n}^{M}=\delta^{M^{2}}+\frac{(2 n+1)^{2} \pi^{2}}{s_{f}^{2}} \\
A^{M}=\Lambda^{2} \frac{\left(1-\exp \left(-\delta^{M} s_{f}\right)\right)}{2 \sinh \left(\delta^{M} s_{f}\right)}, \quad B^{M}=\frac{\Lambda^{2}\left(\exp \left(\delta^{M} s_{f}\right)-1\right)}{2 \sinh \left(\delta^{M} s_{f}\right)}
\end{gathered}
$$

Eqs. (3.1) and (3.2) were solved numerically for the initial boundary value problem (3.3) and (3.8). First the indicated differentiations in (3.1) and (3.2) were performed so that all derivatives appeared explicitly. The derivatives were then replaced by centered difference quotients, i.e.,

$$
\begin{aligned}
x^{\prime} \rightarrow \frac{x_{m+1, n}-x_{m-1, n}}{2}, & r^{\prime} \rightarrow \frac{r_{m+1, n}-r_{m-1, n}}{2 \Delta s}, \\
x^{\prime \prime} \rightarrow \frac{x_{m+1, n}-2 x_{m, n}+x_{m-1, n}}{\Delta s^{2}}, & r^{\prime \prime} \rightarrow \frac{r_{m+1, n}-2 r_{m, n}+r_{m-1, n}}{\Delta s^{2}}, \\
x_{t t} \rightarrow \frac{x_{m, n+1}-2 x_{m, n}+x_{m, n-1}}{\Delta t^{2}}, & r_{t t} \rightarrow \frac{r_{m, n+1}-2 r_{m, n}+r_{m, n-1}}{\Delta t^{2}} .
\end{aligned}
$$

The resulting algebraic equations were then solved for $x_{m, n+1}$ and $r_{m, n+1}$. This is made particularly easy by the fact that $x_{t}$ and $r_{t}$ appear linearly in the differential equations. The values of $x_{m, 0}, r_{m, 0}, x_{m, 1}$ and $r_{m, 1}$ for $0 \leq m \leq \mu$ (if $0 \leq s \leq s_{f}$ is divided into $\mu$ intervals) are determined from the initial conditions. Thus $x_{m, 0}=\sigma m \Delta s, r_{m, 0}=a$, and $x_{m, 1}=x_{m, 0}, r_{m, 1}=r_{m, 0}$ for $0 \leq m \leq \mu$ since $x_{t}(s, 0)=r_{t}(s, 0)=0$. The values of $x_{0, n}, r_{0, n}$ and $x_{\mu, n}, r_{\mu, n}$ are fixed by the boundary conditions (3.8). Thus $x_{0, n}=0$, $r_{0, n}=a, x_{\mu, n}=\sigma s_{f}$, and $r_{\mu, n}=a$ for all $n \geq 0$. It has been pointed out above that previous authors have had difficulty in solving membrane problems for small strains. The present situation is no exception. If $\sigma$ is close to 1 (i.e. small strains) the numerical scheme does not converge unless $\Delta t$ is chosen so small as to make the computing time required prohibitive. However, as $\sigma$ increases it is possible to choose $\Delta t$ larger and larger and still have a convergent scheme. It was found that if the cylinder was stretched to one and a half times its unstrained length, so that $\sigma=1.5$, it was possible to obtain a convergent scheme, and at the same time choose $\Delta t$ sufficiently large that it was possible to obtain numerical solutions for large $t$ in a reasonable amount of computer time. In fact, solutions were found up to the point of maximum deflection of the membrane. At this point the velocity changes sign (the membrane ceases to fall and begins to rise); this change in sign of the velocity definitely affects the convergence of the scheme and the time required to integrate beyond this point is prohibitively large. 


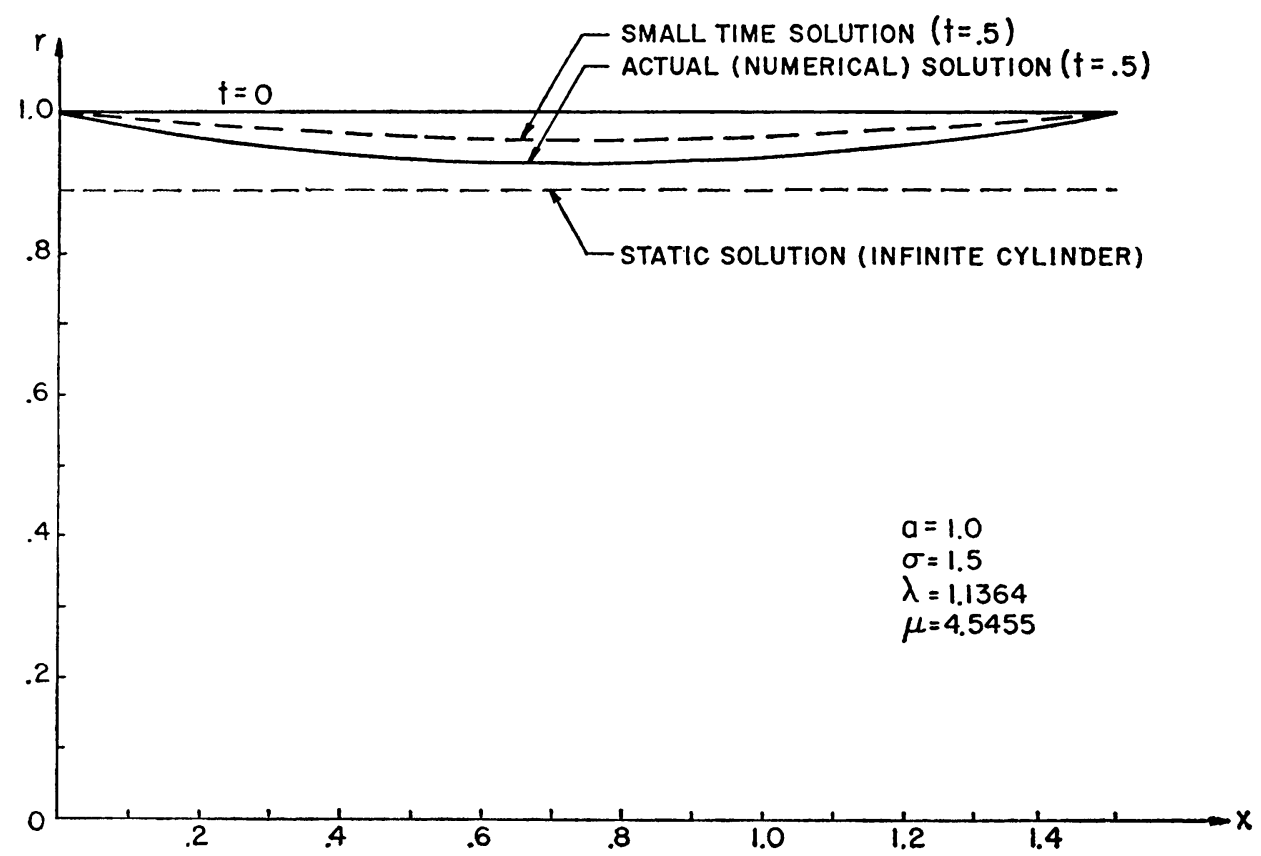

Frg. 3.1. Classical materials

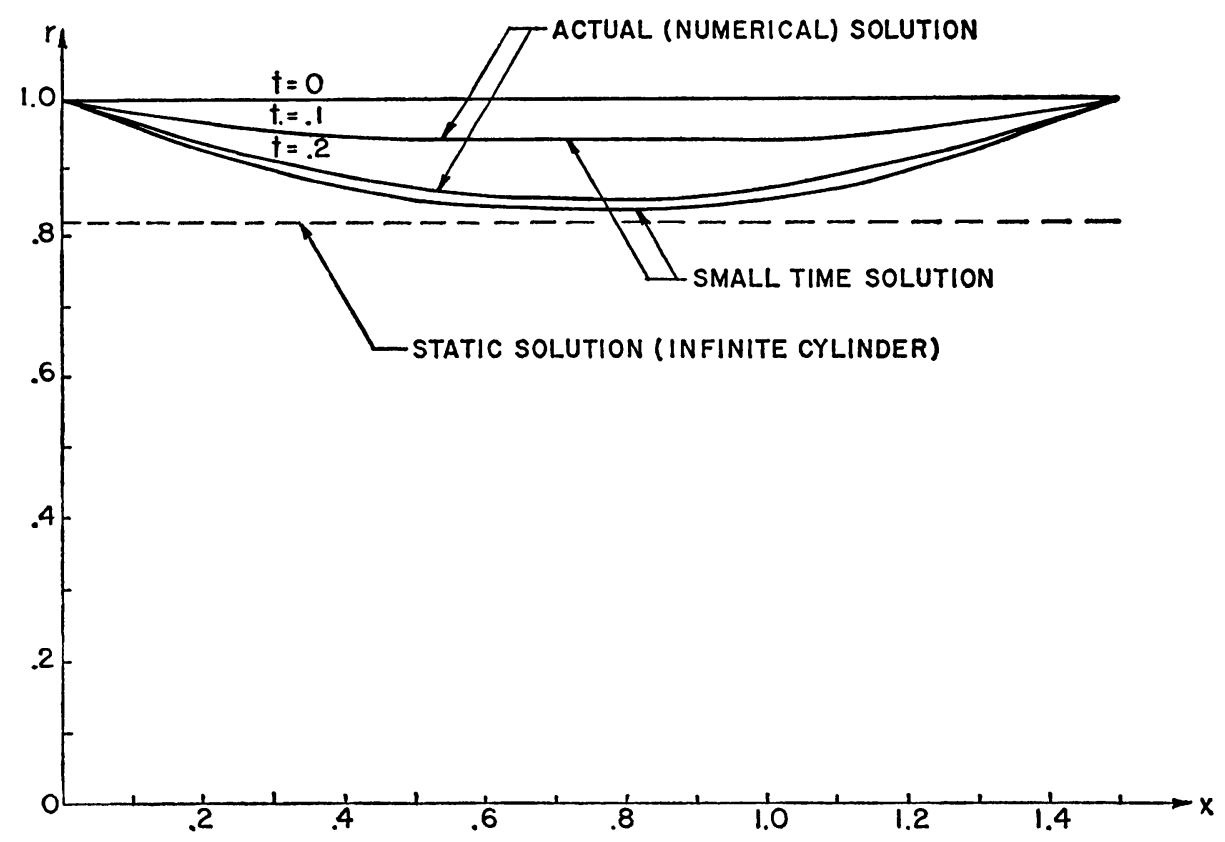

FIG. 3.2. Mooney materials 
The numerical solution of (3.1) and (3.2) is shown in Fig. 3.1 and Fig. 3.2 with $\sigma=$ 1.5. For classical materials $\lambda, \mu$ and $\rho$ were chosen as $\lambda=1.1364, \mu=4.5445$, and $\rho=1$. These values of $\lambda$ and $\mu$ are much smaller than they would be for a realistic material. However, the magnitudes of $\lambda$ and $\mu$ do not affect the form of the surface but only the speed at which the surface reacts. The equations (3.2) for Mooney materials were divided by $C_{2}$ and the ratios $C_{1} / C_{2}$ and $\rho / C_{2}$ were chosen as $C_{1} / C_{2}=9$ and $\rho / C_{2}=1$. The same comments hold here as for classical materials. In Fig. 3.1 the membrane reaches its maximum deflection at $t=.5$ and in Fig. 3.2 the maximum deflection occurs at $t=.2$. In these figures the small time solution is compared to the actual numerical solution. The agreement is seen to be very good throughout the total motion depicted, in fact in Fig. 3.2 the small time solution and the numerical solution overlap at $t=.1$.

\section{REFERENCES}

[1] R. W. Dickey, Dynamic behavior of soap films, Quart. Appl. Math. (2) 24, 97-106 (1966)

[2] J. J. Stoker, Elastic deformation of thin cylindrical sheets, Prager Anniversary Volume, Progress in Applied Mathematics, Macmillan, New York, 1962

[3] A. Corneliussen and R. Shield, Finite deformation of elastic membranes with application to the stability of an inflated and extended tube, Arch. Rational Mech. Anal. (4) 7, 273-304 (1961)

[4] R. Courant and D. Hilbert, Methods of mathematical physics, Vol. I (first English ed.), Interscience, New York, 1953

[5] R. Courant and D. Hilbert, Methods of mathematical physics, Vol. II (first English ed.), Interscience, New York, 1962

[6] A. Bitzadse, Equations of mixed type, Macmillan, New York, 1964 